\title{
UN MODELO DE ECUACIONES SIMULTANEAS DEL EMPLEO Y SALARIOS EN LA INDUSTRIA MANUFACTURERA MEXICANA
}

\author{
Jonathàn KIng \\ Instituto Politécnico Nacional
}

El PROPÓsito de ESTE trabajo es presentar los resultados de un análisis econométrico del sector manutacturero mexicano.' Dadas las limit. . ciones en la disponibilidad de datos trimestrales, de la categoría "resto de la economía" únicamente se pudo especificar al sector monetario, que impacta sobre el sector manufacturero. Las demás interacciones son implícitas y no modeladas. IJas interacciones básicas del modelo se ilustran en la Figura 1.

Con datos trimestrales del período $1970-78$ se estimaron funciones estadísticas explicativas, para el sector manufacturero, del número de personas ocupadas, las horas-hombre trabajadas, la tasa salarial por hora-hombre, y del producto real. Para el sector monetario se explica la variación en el índice nacional de precios al consumidor. La base teórica del estudio es la teoría microeconómica de la empresa y la teoría monetaria de los precios. Para propósitos de comparación, las especificaciones econométricas de las ecuaciones de empleo se basan en las usadas en el modelo Wharton reportado por Evans, y en las del estudio de Black y Kelejian. ${ }^{2}$

\footnotetext{
${ }^{1}$ La lista de actividades que integran el sector manufacturero se encuentra en la parte posterior de este trabajo.

2 Véase a Michael Ẹvans, Macroeconomic Activity, New York, Harper \& Row 1969 , págs. 259-60; y S. W. Black and H. H. Kelejian, "A Macro Model of the U. S. Labor Market", Econometrica, September 1970, págs. 712-40. Un resumen breve sobre modelos de empleo basados en series de tiempo se encuentra en M. Ishaq Nadiri and Sherwin Rosen, A Disequilibrium Model of Demand for Factors of Production, New York, NBER-Columbia. 1974, págs. 2-5.
} 
La estimación econométrica se llevó a cabo con cuatro técnicas: Cuadrados Mínimos Ordinarios (OLS), la Técnica Iterativa de Coichrane y Orcutt (CO), Cuadrados Mínimos de Dos Etapas (TSLS), y la Técnica Iterativa con Variables Instrumentales (TSCO). Las primeras dos técnicas normalmente son consideradas como técnicas de estimación en el contexto de modelos de una ecuación únicamente, donde supuestamente no existe la simultaneidad. Si ciertos supuestos sobre el término de error son válidos (e.g., ausencia de autocorrelación) entonces OLS produce estimadores de coeficientes que son eficientes (i.e., de varianza mínima). Pero cuando existe evidencia de autocorrelación, la técnica $\mathrm{CO}$ produce estimadores más eficientes. En este trabajo se intentó detectar la autocorrelación con la ayuda del estadístico Durbin-Watson.

En el contexto de ecuaciones simultáneas, OLS ya no produce estimadores consistentes (i.e., estimadores cuyo sesgo disminuye y cuya eficiencia aumenta al incrementar el tamaño de la muestra), pero los estimadores TSLS sí tienen esta propiedad. El lector notará que algunas de las ecuaciones de un sistema simultáneo se han estimado también por medio de OLS. Esto se justifica porque OLS produce estimadores más eficientes que TSLS y podría darse el caso de que si el objetivo es minimizar el error cuadrado medio (i.e., varianza más sesgo al cuadrado) de los estimadores, OLS es superior. También OLS podría ser preferible bajo criterios de predicción. En todo caso, se pueden comparar las estimaciones generadas por estas dos técnicas para determinar si la imposición de una estructura de simultaneidad cambia los resultados de estimación de una manera importante. Por último, en presencia de autocorrelación la técnica TSCO produce estimadores también consistentes pero más eficientes que TSLS.

Con el propósito de realizar pruebas de hipótesis sobre los coeficientes estimados, se ha supuesto que la distribución del término de error es, dependiendo del caso, normal o lognormal (i.e., el logaritmo del error tiene una distribución normal). No se realizaron pruebas de hipótesis para detectar la heteroeskedasticidad (i.e., varianza inconstante del término de error) ni para probar la linealidad del modelo.

Finalmente, se optó por utilizar una variante de las ecuaciones explicativas de las variaciones en el número de personas ocupadas para el propósito de proyectar los incrementos en el empleo manufacturero hasta el cuarto trimestre de 1982 y 1985, respectivamente. Estas proyecciones se realizaron tomando en cuenta valores alternativos del crecimiento posible de la producción manufacturera 
real y la tasa real de salarios en este sector, que son las principales variables independientes de la ecuación predictiva, desarrollándose así distintos escenarios del crecimiento del empleo.

\section{Aspectos teóricos}

El modelo consiste de dos sistemas. En el primer sistema tenemos una ecuación explicativa de la demanda de trabajo para el sector manufacturero en términos de personas ocupadas, una ecuación de salarios nominales por persona ocupada en este sector, una ecuación del producto real manufacturero, y una ecuación explicando el índice nacional de precios al consumidor. ${ }^{3}$ El segundo sistema es idéntico al primero con la excepción de que la demanda de trabajo es en términos de horas hombre y los salarios nominales también se especifican por hora-hombre, ambos en lugar de por personas ocupadas. Las dos últimas funciones pertenecen a ambos sistemas. A continuación se plantea el modelo, indicándose la tabla correspondiente para encontrar las estimaciones respectivas. Los símbolos utilizados se explican al final del trabajo.

Sistema I

Tabla

(1) $\quad \mathrm{EM}=\mathrm{f}(\mathrm{RWME}, \mathrm{RWMELAG1,} \mathrm{RRQM,} \mathrm{RRQMLAG1,}$ RRQMLAG2, T)

(2) NWME $=\mathrm{g}(\mathrm{AQEM}$, AQEMLAG1, PCNLAG1, PCNLAG2, PCNLAG3, T, B2 a B11)

Sistema II

(3) $\mathrm{HM}=\mathrm{h}(\mathrm{RWMH}, \mathrm{RRQM}, \mathrm{RRQMLAG1,} \mathrm{RRQMLAG2,}$ T, CUC)

(4) NWMH $=\mathrm{i}(\mathrm{AQHM}$, AQHMLAG1, PCNLAG1, PCNLAG2, PCNLAG2,T, B2 a B11)

Ecuaciones pertenecientes a ambos sistemas

(5) $\quad$ RRQM $=$ j(RCRM, RMLAG1)

3 No existe índice de precios de mayoreo a nivel nacional (únicamente existe para la Ciudad de México) y por lo tanto se ha utilizado el índice nacional de precios al consumidor como deflactor en la construcción de todas las variables reales de este trabajo). 
(6) $\quad$ PCN $=$ k(MLAG1, PCNLAG1, PCNLAG2, T) ( $f, g, h, i, j, k$ son operadores funcionales)

\section{Ecuaciones definicionales (identidades)}

(7) RWME $=$ NWME/PCN

(8) $\quad$ RWMH $=$ NWMH/PCN

(9) NWME $=$ S/EM

(10) $\mathrm{NWMH}=\mathrm{S} / \mathrm{HM}$

(11) $\mathbf{A Q E M}=\mathbf{Q M} / \mathbf{E M}$

(12) $\mathbf{A Q H M}=\mathbf{Q M} / \mathrm{HM}$

(13) $\quad$ RRQM $=$ QM/PCN

(14) $\mathbf{R C R M}=\mathbf{C R M} / \mathrm{PCN}$

(15) $\mathbf{R M}=\mathrm{M} / \mathrm{PCN}$

Las estimaciones econométricas de (1) al (6) se realizaron con distintas especificaciones. La mayoría de las especificaciones son lineales, pero también se estimaron (1) y (3) con especificación logarítmica. También varían las especificaciones en términos de presencia de variables, ya que en algunos casos se supone que los coeficientes de ciertas variables incluidas en la descripción anterior, asumen valores cero. Obviamente no se estimaron las ecuaciones definicionales, pero éstas forman parte del modelo, ya que las dependencias entre variables $\mathrm{y}$, por lo tanto, la simultaneidad, se obtienen también a través de identidades y no solamente por medio de relaciones funcionales.

En la Figura 2 aparece un diagrama de flujo del Sistema I. Las variables con óvalo se consideran como predeterminadas (exógenas o endógenas con retraso) al sistema y aparecen como variables instrumentales en las estimaciones TSLS y TSCO. Las variables encuadradas sin óvalo son las endógenas, explicadas funcionalmente o por medio de identidades. Las rayas gruesas con la flecha en un solo sentido, conectan variables independientes (en la cola de la flecha) con variables dependientes (en la punta). Las rayas delgadas con doble flecha conectan variables relacionadas a través de una identidad. Las rayas punteadas conectan variables con sus versiones retrasadas. Las variables sin raya conectiva (AQHMLAG1, G, Y CUC) son partes de otros sistemas y se utilizan en este sistema únicamente como variables instrumentales. AQHMLAG1 y CUC son variables que pertenecen al Sistema II, y G pertenece al sector "resto de la economía" e implícitamente afecta a este 
sistema. El diagrama de flujo para el Sistema II es muy parecido y se omite.

Ahora procederemos a la derivación teórica de las relaciones funcionales de estos sistemas. Se nota que en las ecuaciones de empleo (1) y (3), las principales variables independientes son el salario real, la producción real y el tiempo, y en (3) también aparece el índice de capacidad. El salario real y la producción real aparecen ambas con estructuras de retrasos; la razón de esto se explicará después de desarrollar la teoría estática de las relaciones funcionales.

Las ecuaciones (1) y (3) son ecuaciones de demanda de trabajo. Por lo tanto, pertenecen a un sistema de ecuaciones de demanda para insumos. Para propósitos de derivación teórica, supondremos la existencia de otros dos factores. La teoría microeconómica de demanda de factores se puede expresar planteando una empresa hipotética que minimiza una función de costos sujeta a una función de producción, con el producto fijo. Utilizando símbolos simples, esto se puede expresar como:

(i) $\operatorname{minimizar} \mathrm{C}=(\mathrm{w} / \mathrm{p}) \mathrm{L}+\mathrm{r}_{1} \mathrm{~K}+\mathrm{r}_{2} \mathrm{~N}$

(ii) sujeta a $Q=q(L, K, N)$

donde se representa costos, $w$ el salario nominal, $p$ el índice de precios, $L$ el insumo trabajo, $\mathbf{r}_{1}$ el precio real del capital, $K$ el capital, $\mathbf{r}_{2}$ el precio real de la tierra, $\mathrm{N}$ el insumo tierra, y $\mathrm{Q}$ el producto. ( $q$ es un operador funcional).

Obteniendo las condiciones de primer orden y solucionando, tenemos las funciones de demanda para los tres factores. ${ }^{4}$ La ecuación de demanda para el trabajo es:

\section{(iii) $\left.\mathrm{L}=\mathrm{l}(\mathrm{w} / \mathrm{p}), \mathrm{r}_{1}, \mathrm{r}_{2}\right) \mathbf{Q}$ (l es un operador funcional)}

En esta ecuación, vemos que la demanda de trabajo depende del salario real, los precios reales de los otros factores y del producto. Desgraciadamente, no tenemos datos sobre $r_{1}$ y $\mathbf{r}_{2}$.

Conviene, en esta parte, introducir una cuestión de tipo econométrico. En lugar de cometer un serio error de especificación omitiendo estos precios, recurrimos a la sustitución de una variable

4 Véase P.R.G. Layard and A.A. Walters, Microeconomic Theory, New York, Mc Graw-Hill, 1078, pág. 268. 
"Proxy" bajo el supuesto de que las variables omitidas están correlacionadas con el "proxy" que, en este caso, es T, el tiempo. Introducción a $\mathrm{T}$ en lugar de $\mathrm{r}_{1}$ y $\mathrm{r}_{2}$ en (iii) tenemos:

(iv) $\mathrm{L}=\mathrm{F}(\mathrm{w} / \mathrm{p}, \mathrm{T}) \mathrm{Q}$

(F es un operador funcional)

donde la derivada parcial de $\mathrm{L}$ con respecto al salario real es negativa, la derivada parcial de $\mathrm{L}$ con respecto a $\mathbf{Q}$ es positiva y el signo de la derivada parcial de $\mathrm{L}$ con respecto a $\mathrm{T}$ es incierta. ${ }^{5}$ Esto justifica la inclusión del salario real, el producto real y el tiempo en las ecuaciones econométricas de demanda de trabajo y nos da una idea de los signos esperados de los coeficientes.

Ahora bien, en la relación (3) también se ha incluido como variable independiente a CUC, el coeficiente de utilización de capacidad productiva. Según Evans, en tiempos de expansión cíclica las empresas tienen dificultad en contratar el personal adicional que desean y se ven obligadas a recurrir a horas extras. Por lo tanto, la demanda de horas-hombre está en función de la utilización de la capacidad. ${ }^{6}$

Pasando ahora a las funciones (2) y (4) que explican los salarios nominales, podemos transformar a (iv) para obtener la relación teórica deseada:

(v) $1 / F(w / p, T)=Q / L$

dejando que $G(w / p, T)=1 / F(w / p, T)(G$ es un operador funcional)

entonces:

(vi) $\mathrm{G}(\mathrm{w} / \mathrm{p}, \mathrm{T})=\mathrm{Q} / \mathrm{L}$

5 Al parecer, se podría afirmar que los precios de los otros factores posiblemente estén positivamente correlacionados con $\mathrm{T}$, el tiempo. Al aumentar el ingreso real a través del tiempo, es lógico suponer que los factores de producción responsables de este aumento reciban el beneficio correspondiente. Teóricamente se espera que aumentos en los precios reales de los otros factores produzcan, ceteris paribus, un aumento en la demanda de trabajo. Por lo tanto, al reflejar T los movimientos de estos precios, la derivada parcial de $\mathrm{L}$ con respecto a $\mathrm{T}$ debería ser positiva. Pero hay que observar que $\mathrm{T}$ podría captar los efectos de otras variables también, como es el caso del cambio tecnológico. Se espera que el cambio tecnológico, cateris paribus, reduzca la utilización del trabajo. Por lo tanto, $\mathrm{T}$ refleja ciertos factores que influyen a $\mathrm{L}$ en forma opuesta y esta es la razón por la cual no se anticipa signo específico a su derivada parcial.

${ }^{6}$ Véase a Evans, pág. 259. 
separando a $\mathrm{w} / \mathrm{p}$ tenemos:

(vii) $w / p=H(Q / L, T)$ (H es un operador funcional)

y para salarios nominales:

(viii) $\mathbf{w}=\mathrm{H}(\mathrm{Q} / \mathrm{L}, \mathrm{T}) \mathrm{p}$

donde la derivada parcial de $\mathrm{w}$ con respecto al producto medio es positiva, con respecto a $\mathrm{p}$ es positiva y con respecto a $\mathrm{T}$ es incierta. ${ }^{7}$

Además de la estructura de retrasos de Q/L y p, falta explicar por qué se incluyen en (2) y (4) las variables binarias (mudas) B2 a B11. Estas variables binarias recogen ciertos aspectos de tipo institucional que operan sobre la determinación del salario nominal, además de las fuerzas del mercado. Representan los períodos de tiempo durante los cuales rige un determinado salario mínimo oficial. Generalmente, al aumentarse el salario mínimo, se reajusta hacia arriba toda la estructura salaria, aumentándose así el salario nominal promedio. Por lo tanto, cambios en el salario mínimo oficial, representados a través de estas variables, deberán afectar los salarios nominales.

Pasemos ahora a las ecuaciones (5) y (6). En la (5) vemos que el producto real depende de las balanzas reales de crédito y de las balanzas reales de dinero. Este planteamiento se debe a que la producción manufacturera $Q$ se determina por condiciones de oferta y por condiciones de demanda. Considerando los insumos, es probable que exista exceso de demanda de capital (en formas de créditos bancarios) al costo prevaleciente y que este factor sea el que limita los aumentos en $\mathbf{Q}$ por el lado de la oferta. Por el lado de la demanda, el deseo de producir $\mathbf{Q}$ está en función del ingreso agregado. Como no tenemos datos para medir el ingreso real agregado, necesitamos encontrar un "proxy". Para lograr esto, aprovechemos la teoría cuantitativa del dinero, expresada a través de la siguiente ecuación:

(ix) $(\mathrm{M} / \mathrm{p}) \mathrm{V}=\mathrm{Y}$

\footnotetext{
7 La ecuación teórica (viii) expresa a w como función de $Q / L$. Esta $Q$ correspondería a nuestra variable observada RRQM, o sea el producto real manufacturero. Pero en las ecuaciones (2) y (4) vemos que el salario nominal está en función del producto medio nominal y no del producto medio real. Esto se debe a que se ha optado por separar la influencia de los precios del otro componente del producto medio real, que es el producto medio nominal. I,a influencia de los precios se percibe con una estructura de retrasos.
} 
donde $\mathrm{M}$ es la oferta monetaria, $\mathrm{V}$ es la velocidad de circulación del dinero, e $\mathrm{Y}$ es el producto real (ingreso real agregado). En la teoría cuantitativa $\mathrm{V}$ es una función de diversas variables, entre ellas la tasa de interés. Al aumentar $Y$ es probable que aumente $(M / p)$ y además $\mathrm{V}$. Por lo tanto $(\mathrm{M} / \mathrm{p})$ estará correlacionada con $\mathrm{Y}, \mathrm{y}$ sirve de "proxy" para dicha variable. Esto explica la especificación de la ecuación (5) en cuanto a variables independientes. ${ }^{8}(\mathrm{M} / \mathrm{p})$ aparece retrasada porque los aumentos en la demanda requieren tiempo para traducirse en aumentos en la producción.

En la ecuación (6) el índice de precios se explica por la oferta monetaria, el tiempo, e índices de precios retrasados. La presencia de los índices retrasados se justificará en seguida $\mathrm{y}$, por ahora, veamos por qué se incluyen las primeras dos variables. Transformando a (ix) tenemos:

$$
\mathrm{p}=\mathrm{M}(\mathrm{V} / \mathrm{Y})
$$

En esta forma, (x) es una identidad y no tiene sentido hablar de una estimación econométrica para ella. Pero podemos introducir los determinantes de $\mathrm{V}$ y establecer una relación funcional. Se considera a $\mathrm{V}$ como determinado por ingresos reales $\mathrm{Y}$ y por los costos de mantener balanzas reales. Siguiendo a Vogel:

(xi) $\quad \mathrm{v}^{-1}=\mathrm{Y}^{\mathrm{a}} \mathrm{C}^{\mathrm{b}}$

donde la proporción del ingreso real mantenido como balanzas reales, i.e., la inversa de $\mathrm{V}$, depende del ingreso $\mathrm{Y}$ y los costos esperados de mantener estas balanzas C. (a y b son parámetros). ${ }^{9}$ Por lo tanto:

$$
\mathrm{p}=\mathrm{MY}^{\mathrm{a}-1} \mathrm{C}^{\mathrm{b}}
$$

Si $Y^{a^{-1}} C^{-b}$ está correlacionada con $T$, el tiempo, esta última se puede

8 Es interesante comparar esta especificación con la mencionada por Evans para el Modelo Wharton. Ahí, el producto real manufacturero está en función de las siguientes variables: gastos reales sobre bienes de consumo no duraderos y servicios, gastos reales sobre bienes de consumo duraderos, inversión real en planta y equipo no agrícola, el cambio en el acervo de inventarios no agrícolas, y los gastos reales del gobierno para la defensa nacional. Este planteamiento considera únicamente a los integrantes de la demanda como determinantes de la producción manufacturera. En nuestra especificación no se logra desagregar la demanda, pero sí se considera que también se determina el producto por el lado de la oferta. Véase a Evans, pág. 437.

9 Véase a R. C. Vogel, "The Dynamics of Inflation in Latin America, 1950 1969". American Economic Review, March 1974, pág. 107. 
utilizar como "proxy" en el modelo. M se incluye en forma retrasada en (6) porque se piensa que el impacto de aumentos en la oferta monetaria sobre los precios no es instantánea.

Por último, se explicará la presencia de retrasos en las ecuaciones. Vimos que en las ecuaciones (5) y (6) la oferta monetaria real y nominal, respectivamente, aparecían con retraso de un período para reflejar el impacto no instantáneo de los cambios representados por esta variable. Pero éste es únicamente un motivo entre varios por el cual se introducen retrasos. Otro motivo es intentar captar el fenómeno de expectativas adaptativas, en donde una variable dependiente está en función del valor esperado, más que del valor actual, de una variable independiente. Por ejemplo, en las ecuaciones (2) y (4); los salarios nominales están determinados por las expectativas acerca de los precios y la productividad. Como los salarios nominales no se alteran en forma continua sino que se fijan por períodos, cualquier cambio se realiza tomando en cuenta lo que se espera en el futuro no muy lejano. Estas expectativas del futuro están en parte determinadas por las tendencias del pasado, y éstas se reflejan en los valores retrasados de las variables en cuestión.

Se podría afirmar algo muy similar para las ecuaciones (1) y (3). El nivel de empleo actual no es necesariamente el óptimo dados los salarios y el producto actual. Por ejemplo, si se espera producir más en el futuro, con mayor cantidad de mano de obra, se podría contratar ésta en el presente para cumplir con los planes futuros de producción sin dificultad. Las expectativas hacia el futuro se pueden captar a través de una estructura de retrasos en las variables independientes de salario real y producción.

La explicación para la ecuación (6) es un poco más complicada. Podemos suponer que existe un valor esperado de la oferta monetaria y un valor deseado del índice de precios. Esto representa un modelo con el fenómeno de expectativas adaptivas para la oferta monetaria y con el fenómeno de ajuste parcial para el índice de precios. Si la sociedad deseara un determinado nivel del índice de precios, el nivel actual representa un paso hacia el logro del nivel deseado (o sea, que el ajuste hacia el nivel deseado es parcial dentro de determinado período). La estructura de (6) es consistente con esta racionalización, aunque su estimación con cuadrados mínimos ordinarios produce estimadores inconsistentes. Por lo tanto, se estimaron también relaciones más simples sin los precios retrasados. ${ }^{10}$

10 Véase a Jan Kmenta, Elements of Econometrics, New York, Macmillan, 1971, págs. 477-79, para una explicación detallada de este problema. 


\section{Figura 1}

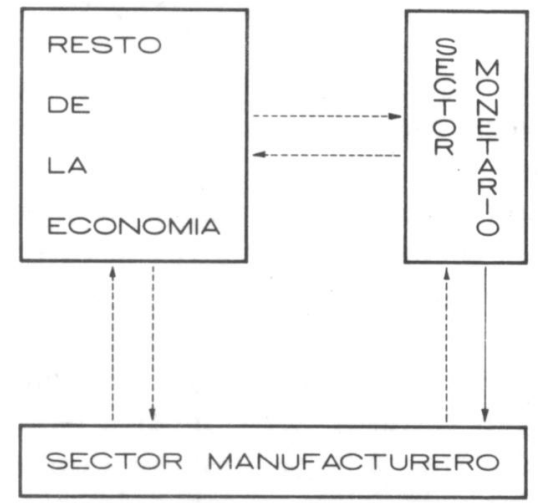

Relación entre el Sector Manufacturero, el Sector monetario y "El Resto de la Economía". La raya continua indica una determinación explícita del Sector Manufacturero por el Sector Monetario. Las rayas punteadas indican relaciones implícitas no modeladas.

\section{Figura 2}

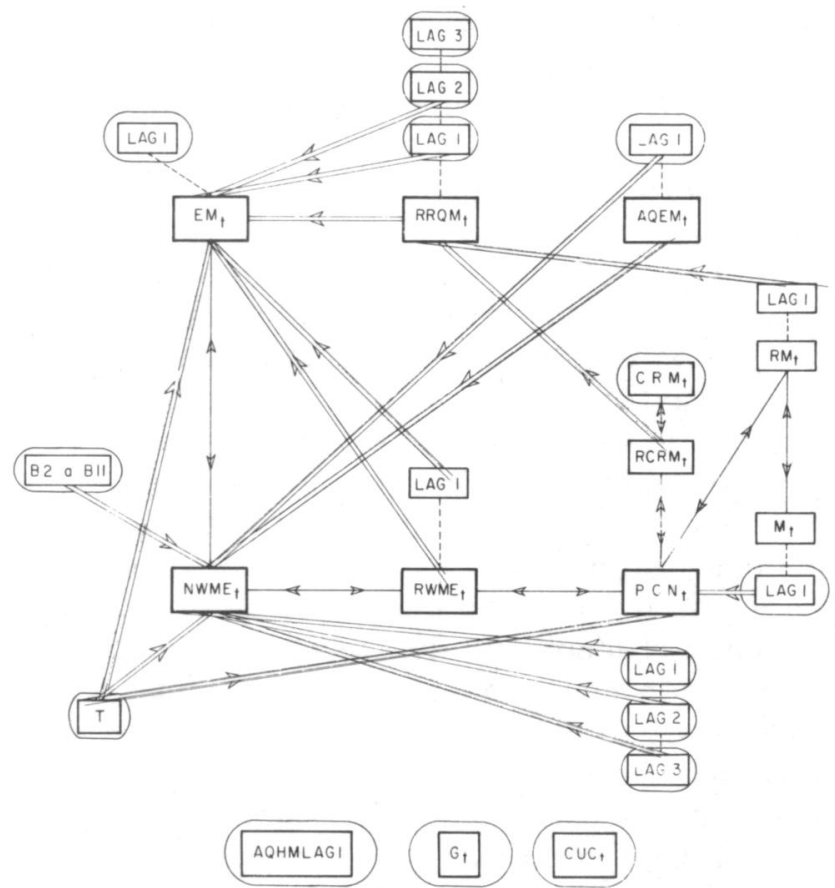

Diagrama de flujo para el Sistema I. Las variables con óvalo son las predeterminadas. Las rayas gruesas con la flecha en un sentido unen a las variables dependientes (en la punta) con variables independientes en relaciones funcionales. Las rayas delgadas con doble flecha indican una relación definicional entre variables. Las rayas punteadas indican retrasos de las variables respectivas. Las variables con óvalo pero sin raya únicamente aparecen como variables instrumentales en las estimaciones TSLS y TSCO. 


\section{Los resultados: demanda de trabajo}

En las tablas I-IV se presentan varias estimaciones de ecuaciones de empleo. Las variables dependientes son, según el caso, el empleo medido como número de personas ocupadas (Tablas I y III) o como horas-hombre (Tablas II y IV). Las estimaciones lineales se presentan en las Tablas I y II y las logarítmicas en las Tablas III y IV. ${ }^{1}$ Se espera que las variables que miden el producto real tengan coeficientes con signo positivo y las que miden el salario real tengan coeficientes con signo negativo. Se espera que el coeficiente de la variable de utilización de capacidad tenga signo positivo y que el coeficiente de la variable tiempo tenga cualquier signo.

En las tablas I y II se presentan resultados de regresiones estimadas por la técnica de cuadrados mínimos ordinarios (OLS). Uno de los supuestos para su aplicación correcta es la ausencia de autocorrelación. Pero debido a los valores relativamente bajos del estadístico Durbin-Watson, se reestimaron utilizando la técnica iterativa de Cochrane-Orcutt (CO). En total se reportan, en estas dos tablas, scis ecuaciones del empleo en términos de número de personas ccupadas y dos del empleo en términos de horas-hombre. En las tablas III y IV se presentan regresiones estimadas por estas técnicas y además las de cuadrados mínimos de dos etapas (TSLS) y la itera-

11 Para propósitos de comparación, una ecuación reportada por Evans (véase la referencia para el período de estimación y demás detalles) es:

$$
\mathrm{h}_{\mathrm{m}}=0.797+0.00076 \mathrm{X}_{\mathrm{m}}+\underset{(0.00021)}{0.00126 \Delta \mathrm{X}_{\mathrm{m}}+0.1906 \mathrm{C}_{\mathrm{p}}-0.0126 \mathrm{wr}_{\mathrm{m}}}
$$

$R^{-2}=0.711$. Los errores estándar están entre paréntesis.

Los símbolos son:

$\mathrm{h}_{\mathrm{m}}$ ' índice de horas trabajadas por semana, 40 horas $=1 ; \mathrm{X}$, producción bruta de origen en billones de dólares de 1958; $\mathrm{Cp}$, índice porcentual de utilización de capacidad; $w_{m}$, tasa salarial anual, en miles de dólares por año; $m$, manufacturas. Black y Kelejian reportan la siguiente regresión (véase la referencia para los demás detalles):

$\log \mathrm{L}=-.05-.0026 \mathrm{~T}-.364 \log (\mathrm{w} / \mathrm{P})-.557 \log \mathrm{Q}+.223 \log \mathrm{Q}^{-1}+$

$.092 \log Q_{-2}$

$$
\begin{array}{lll}
(-2.0) & (-1.9) \quad(8.3) \quad(5.3)
\end{array}
$$

$\mathrm{R}^{2}=.98$. Valores del estadístico -t se encuentran entre paréntesis. Los símbolos son: $\mathrm{L}$, horas-hombre trabajadas en el sector privado no agrícola, en billones; $\mathrm{T}$, tiempo; (w/P), salario real por hora-hombre en el sector arriba mencionado; $Q$, producto deflactado del sector arriba mencionado, a precios de 1958 . 
tiva de dos etapas (TSCO), que generan estimadores consiștentes. ${ }^{12}$ En estas tablas se reportan cuatro ecuaciones del empleo en términos del número de personas ocupadas e igual número en términos de horas-hombre. Además se realizó un análisis de multicolinealidad para las ecuaciones de las Tablas III y IV.

Desde el punto de vista de los estadísticos que resumen el poder explicativo y predictivo de las regresiones, las ecuaciones estimadas en estas cuatro tablas lucen favorablemente. El valor mínimo encontrado del coeficiente de correlación múltiple corregido por grados de libertad, $\mathrm{R}^{-2}$, es de .936 en la ecuación (III-3) y el valor mínimo del coeficiente de correlación entre la variable dependiente observada y la variable independiente predecida por el modelo, $r$, en las ecuaciones estimadas por OLS es de .97683 en la ecuación (III-1). La ecuación (I-1) tuvo el valor más alto de r: .97820.

En la Tabla I, con ecuaciones lineales explicando el número de personas empleadas, todas las variables tuvieron coeficientes con los signos anticipados. El coeficiente de $\mathrm{T}$, el tiempo, sin el signo específico anticipado, empíricamente tuvo signo positivo y significativo (al 10 por ciento o mejor) en todas las regresiones. La variable $R R Q M$, el producto manufacturero real, tuvo coeficientes significativos en todas las regresiones excepto en la (I-1), estimada por OLS. La variable DIFRRQM, que mide la primera diferencia de RRCM, no tuvo coeficiente significativo en las cuatro regresiones en que aparece, de la (I-1) a la (I-4). El salario manufacturero real por persona, RWME, tuvo coeficientes significativos en todas las regresiones pero DIFRWME, que aparece en las regresiones (I-1) y (I-2), nunca tuvo coeficientes significativos.

En la tabla II aparecen dos ecuaciones lineales que explican el número de horas-hombre trabajadas. Los coeficientes de RRQM fueron significativos y con el signo esperado en las dos regresiones, pero los coeficientes de DIFRRQM aparecieron con signos negativos siendo significativo únicamente en (II-1), estimada por OLS.

\footnotetext{
12 Fair observa que para obtener estimadores consistentes de los coeficientes de una ecuación en la aplicación de la técnica iterativa de dos etapas, se requiere incluir entre los instrumentos las siguientes variables: la variable dependiente se incluye con retraso de un período; las otras variables endógenas que aparecen en la ecuación como variables independientes se incluyen con retraso de un período; las variables predeterminadas (exógenas y endógenas con retraso) que aparecen en la ecuación se incluyen en su forma original y también con retraso de un período. Véase a Ray C. Fair, "The Estimation of Simultaneous Equation Models with Lagged Endogenous Variables and First Order Serially Correlated Errors", Econometrica, May 1970, pág. 508.
} 
Tabla I

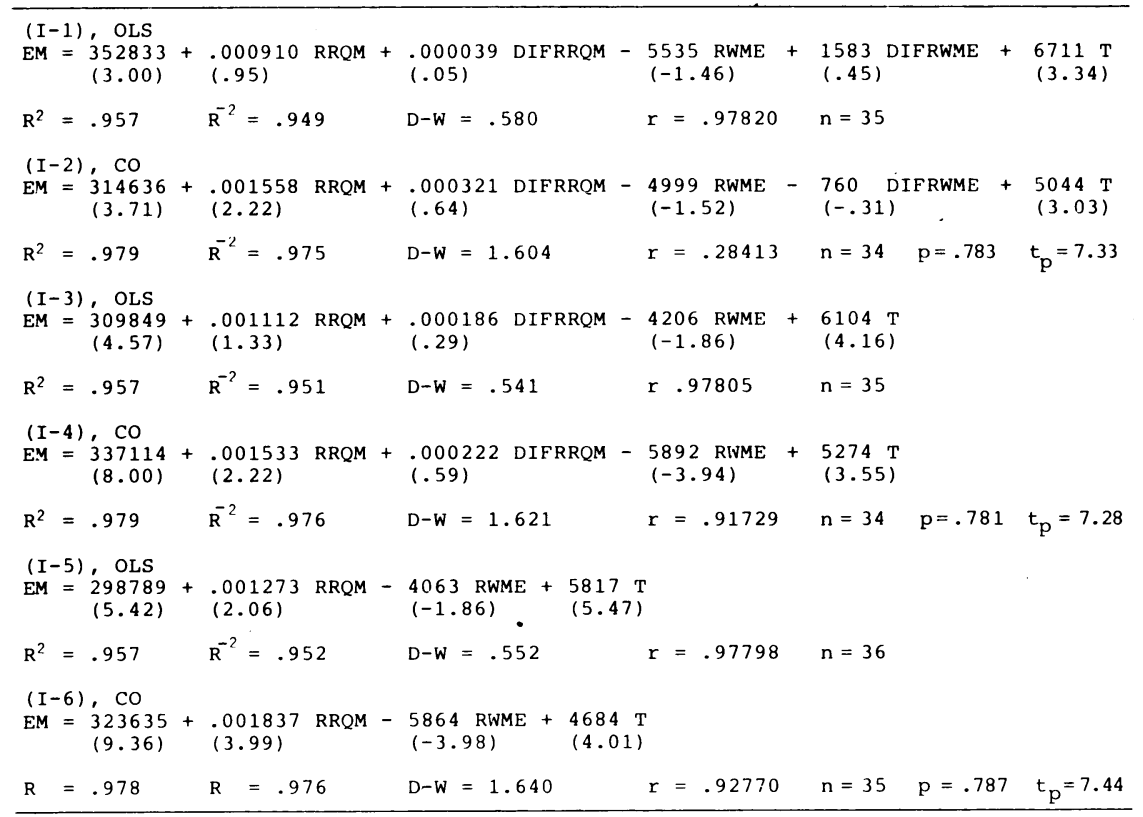

Los coeficientes de RWMH, el salario manufacturero real por horahombre, tuvieron el signo negativo anticipado, pero únicamente el de (II-2), estimado por $\mathrm{CO}$, resultó significativo. Ni la primera diferencia de esta variable ni la variable $\mathrm{T}$ aparecen en estas regresiones, lo que facilitaría la comparación con la regresión reportada por Evans, especialmente en lo referente al comportamiento del coeficiente que mide el grado de utilización de capacidad. En las regresiones de este trabajo, el coeficiente de la variable de utilización de capacidad, CUC, nunca es significativo ni cambia de signo. En contraste, la variable utilizada por Evans tuvo un coeficiente significativo con signo positivo. Esta diferencia de resultado se podría deber, quizás, a que en México el exceso de oferta de trabajo para la industria manufacturera es tán amplio que aún en tiempos de expansión no hay problemas de conseguir personal adicional $\mathrm{y}$ por lo tanto no se ajusta por medio de horas extras, o bien, porque la estimación utilizada en este trabajo no refleja acertadamente las variaciones en la utilización de la capacidad. 
Tabla II

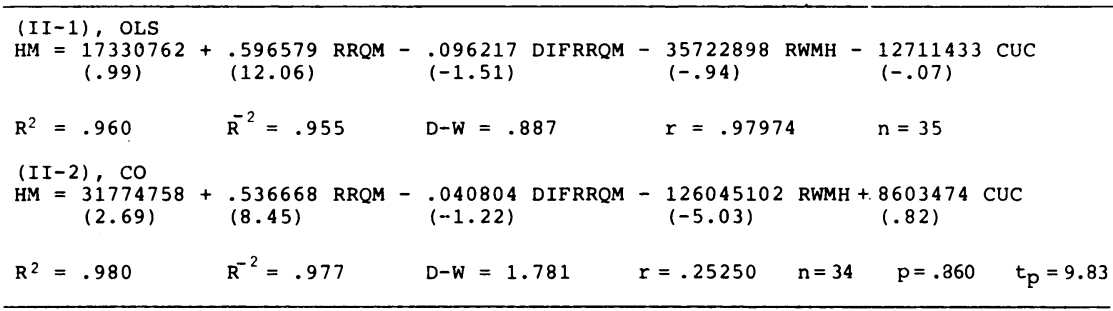

En la Tabla III aparecen cuatro ecuaciones logaritmicas explicando el empleo en términos del logaritmo natural del número de personas ocupadas. Todas las regresiones tienen las mismas variables independientes. Todos los coeficientes tuvieron los signos esperados excepto el de la variable LNRRQMLAG1, que representa el logaritmo natural de la producción real manufacturera retrasada un período, en la ecuación (III-4), estimada por TSCO, pero este coeficiente no fue significativo. Los coeficientes de LNRRQM siempre fueron significativos, así como los de LNRWME, excepto en la regresión (III-3), estimada por TSLS. Los coeficientes de LNRRQMLAG1 y LNRRQMLAG2 nunca fueron significativos, indudablemente, debido al problema de multicolinealidad. En la tabla $\mathrm{V}$ se presentan los coeficientes de correlación múltiple, $\mathrm{R}^{2}$, calculados a partir de las regresiones estimadas por OLS tomando por turno cada variable independiente de las regresiones de la Tabla III, como la variable dependiente, explicada por las demás variables independientes. Todas estas regresiones excepto la de la variable dependiente LNRWME, tuvieron valores de $R^{2}$ por encima de .900 , indicando alta intercorrelación entre las variables independientes.

En la Tabla IV aparecen cuatro ecuaciones logaritmicas, todas con las mismas variables independientes, explicando el empleo en términos de horas-hombre. Todos los coeficientes tuvieron los signos esperados excepto los de LNRRQMLAG2, que únicamente tuvo el signo positivo esperado en (IV-4), estimada por TSCO, pero los coeficientes con signo negativo en las otras regresiones nunca fueron significativos. Los coeficientes de LNRRQM fueron altamente significativos pero la variable LNRRQMLAG1 únicamente tuvo un coeficiente significativo, en la regresión (IV-1), estimada por OLS. Los coeficientes de LNRWMH fueron significativos únicamente en las regresiones (IV-2) y (IV-4). Consultando la Tabla V, de nuevo vemos 
Tabla III

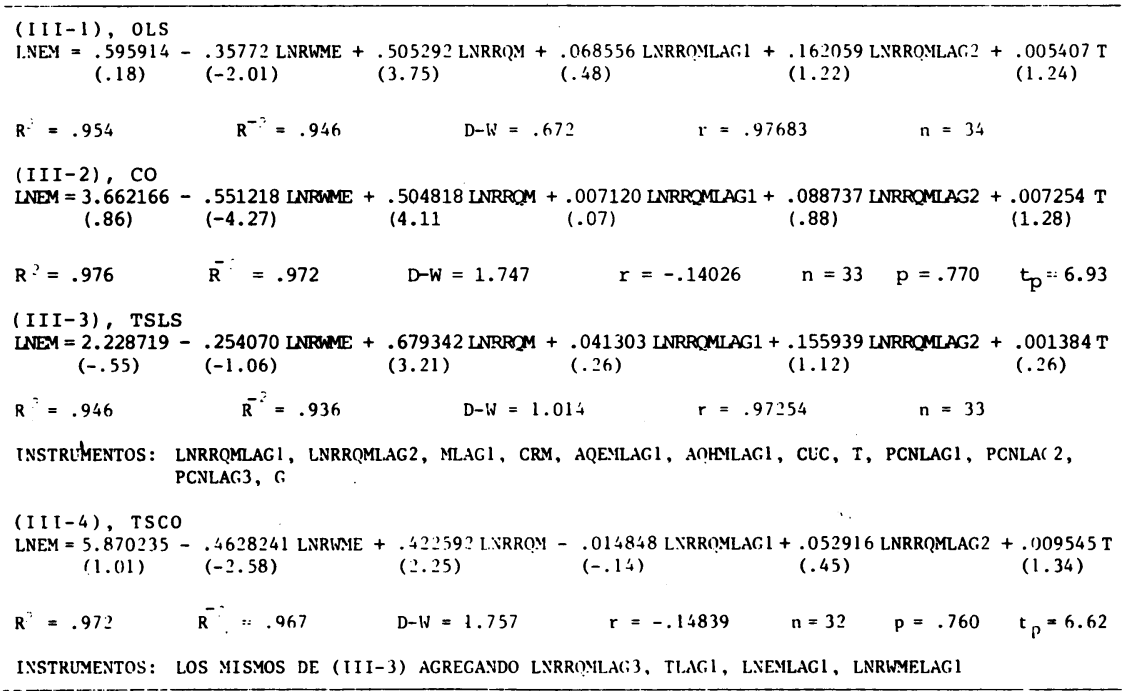

resultados muy parecidos a los obtenidos para las variables de las ecuaciones de la Tabla III. Por lo tanto, al parecer también en estas regresiones la multicolinealidad es la causa de la falta de significación de los coeficientes de las variables con retrasos.

En conclusión, podemos afirmar que las variables del producto real y de la tasa real de salarios por persona o por hora-hombre, en forma lineal o logarítmica, funcionaron generalmente como se esperaba, especialmente en las versiones estimadas por CO o TSCO. La variable $\mathrm{T}$ exhibe un contraste marcaco entre las regresiones de la Tabla I, en comparación con los de la Tabla III. En la Tabla I, se observan coeficientes altamente significativos con signo positivo en todas las regresiones, mientras que en las de la Tabla III los coeficientes también tienen signo positivo, pero significativo únicamente en la (III-4), probablemente, debido a la multicolinealidad que caracteriza a dichas regresiones. La variable CUC no funcionó como se esperaba. Las variables con primeras diferencias o con retrasos, por lo general, no funcionaron como se esperaba, probablemente debido a problemas de multicolinealidad. Las Tablas III y IV revelan que todas las elasticidades de las variables independientes son mayores que uno, en valor absoluto. $\mathrm{Al}$ parecer, los resultodas de estas cuatro tablas no presentan grandes novedades o anomalías, si son considerados como pruebas empíricas de proposiciones teóricas. 


\section{Tabla IV}

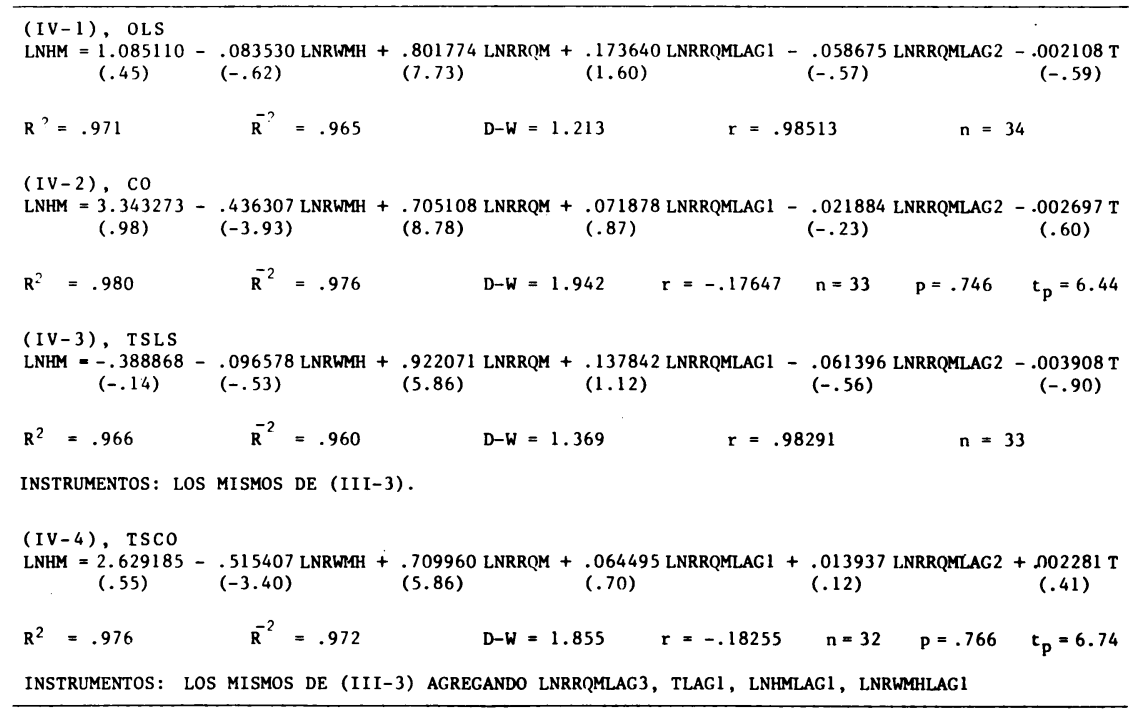

\section{Tabla V} Tabla para el análisis de multicolinearidad. Cada variable independiente
de las tablas II y IV se considera en turno como variable dependiente
explicado en regresiones ols por las variables independientes restantes.

\begin{tabular}{|c|c|c|}
\hline Variable dependiente & $R^{2} \operatorname{con} n=34$ & $\mathrm{R}^{2} \operatorname{con} \mathrm{n}=33$ \\
\hline Variables de la Tabla & & \\
\hline LNRWME & .702 & .683 \\
\hline LNRRQM & .932 & .925 \\
\hline LNRRQMLAG 1 & .938 & .933 \\
\hline LNRRQMLAG 2 & .928 & .922 \\
\hline $\mathrm{T}$ & .964 & .962 \\
\hline \multicolumn{3}{|c|}{ Variablès de la Tabla IV } \\
\hline LNRWMH & .802 & .786 \\
\hline LNRRQM & .936 & .930 \\
\hline LNRRQMLAG 1 & .940 & .936 \\
\hline LNRRQMLAG 2 & .934 & .928 \\
\hline $\mathrm{T}$ & .972 & .969 \\
\hline
\end{tabular}




\section{Los resultados; tasas de salarios nominales}

Los resultados acerca de los salarios nominales se presentan en las ecuaciones de la tabla VI. Las ecuaciones(VI-1) y (VI-2) tienen como variable dependiente de la tasa de salarios nominales por persona, mientras que las ecuaciones (VI-3) y (VI-4) tienen como variable dependiente la tasa de salarios nominales por hora-hombre. Las ecuaciones (V-I) y (VI-3) se estimaron por OLS y las (VI-2) y (VI-4) por TSLS. Se incluyeron en estas regresiones variables de productividad media nominal corriente y con retraso de un período, variables que miden el índice nacional de precios al consumidor con retraso de uno, dos y tres períodos, la variable $\mathrm{T}$ y diez variables binarias (mudas) para capturar el efecto de cambios en los salarios mínimos. Se espera que todas las variables tengan coeficientes con signos positivos, exceptuando $\mathrm{T}$ que puede tener cualquier signo.

Lo sorprendente de los resultados de la Tabla VI son los elevadísimos valores de $\mathrm{R}^{2}, \overline{\mathrm{R}}^{2}$, y r. Todos los $\mathrm{R}^{2}$ fueron del orden de .999 y el $\bar{R}^{2}$ fue de .998 en (VI-1) y (VI-3) y de .997 en las otras dos regresiones. El $\mathrm{r}$ varió de .99955 en (VI-1) a .99910 en (VI-2). Las variables de productividad media corriente, AQEM y AQHM, tuvieron coeficientes con los signos positivos esperados y significativos. AQEMLAG1 tuvo un coeficiente significativo en (VI-2) y positivo y no significativo en las regresiones restantes. AQHMLAG1 tuvo coeficientes que variaron en signo pero no fueron significativos. La variable PCNLAG1, el índice nacional de precios al consumidor retrasado un período, tuvo coeficientes que variaron en signo pero que nunca fueron significativos. PCNLAG2 tuvo coeficientes con signo negativo exceptuando en (VI-1) y significativo únicamente en (VI-3). PCNLAG3 siempre tuvo coeficientes positivos pero únicamente en (VI-1) fue significativo su coeficiente. La variable $\mathrm{T}$ siempre tuvo coeficientes positivos pero fueron significativos únicamente en (VI-1) y (VI-3). El comportamiento errático de los coeficientes de las variables retrasadas, aunado en los $\mathrm{R}^{2}$ tan grandes nos hace sospechar que el problema 'vásico es de multicolinealidad. También hubo comportamiento errático en los coeficientes de las variables binarias, como podrá percatarse el lector al inspeccionar la tabla, pero para las de fechas más recientes tendieron a funcionar como se esperaba.

En conclusión, las ecuaciones de salarios parecen estar bien especificadas, especialmente desde el punto de vista de los resultados estadísticos. Problemas de multicolinealidad, que aparecen típicamente en modelos con retrasos, podrían ser la causa del "comportamiento errático" de muchos de los coeficientes. 
Tabla VI

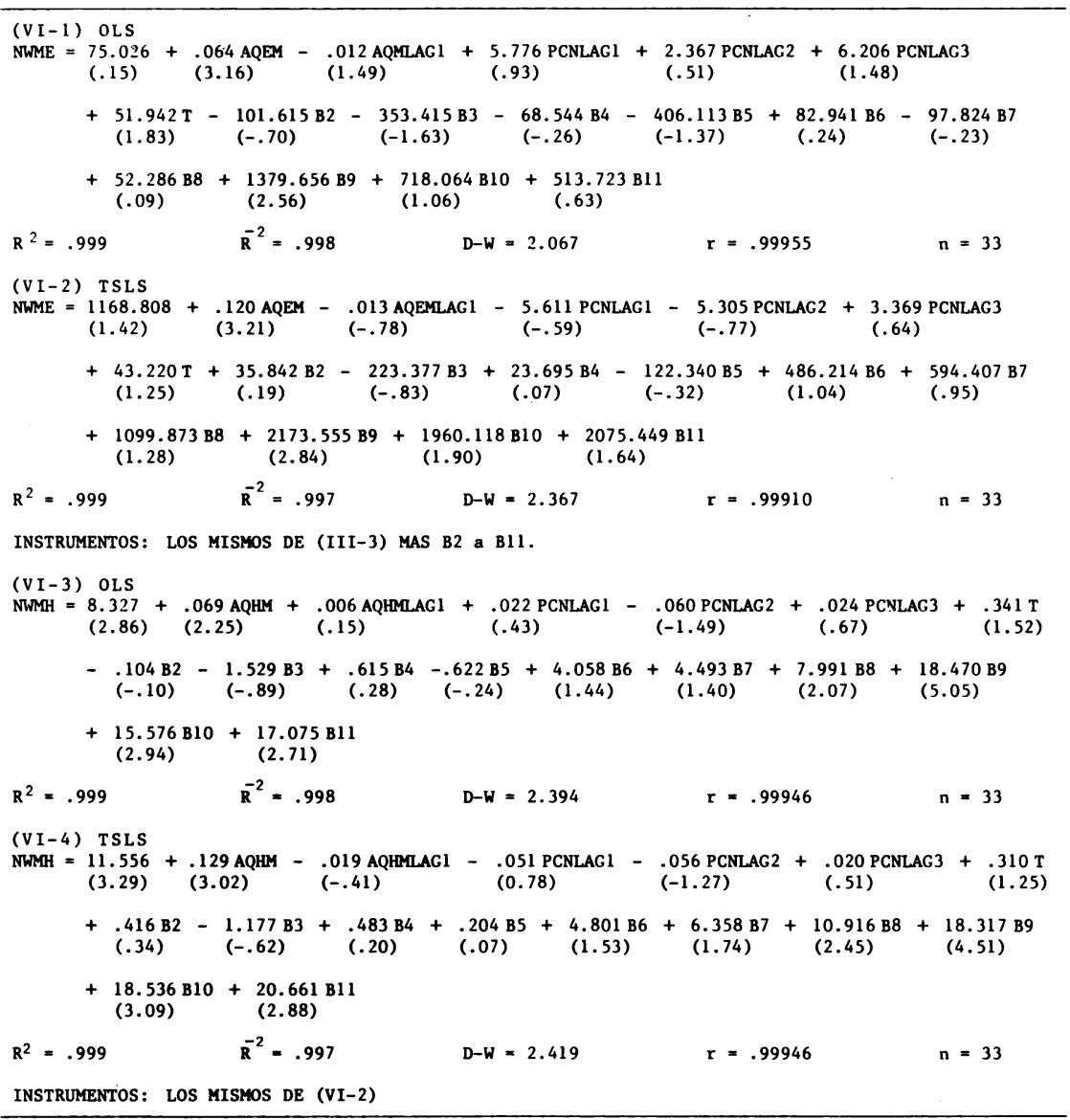

\section{Los resultados: producto real y precios}

En la Tabla VII se observan ecuaciones explicativas de las dos principales variables endógenas que aparecen como independientes en las ecuaciones de empleo y salarios, RRQM y PCN. La ecuación (VII-1) explica RRQM por medio de dos variables independientes: RCRM, los saldos reales de crédito a la industria manufacturera y RMLAG1, la oferta monetaria real con retraso de un período. En la regresión, estas variables tuvieron coeficientes significativos y con el 
signo positivo esperado, aunque el $\mathrm{R}^{2}$ de .884 y $\overline{\mathrm{R}}^{2}$ de .877 fueron los más bajos de todas las ecuaciones aquí reportadas.

Las ecuaciones (VII-2) al (VII-4) explican el índice nacional de precios al consumidor, PCN. En las (VII-2) y (VII-3) las variables independientes son MLAG1 y T. (VII-2) se estimó con OLS y (VII-3) con CO. Las variables independientes resultaron con coeficientes significativos de signo positivo. En la ecuación (VII-4), estimada por OLS, se agregan a estas variables las de PCNLAG1 y PCNLAG2. El cambio de especificación ocasiona que el coeficiente de MLAG1 ya no sea significativo. Los coeficientes de las variables adicionales son de signo positivo pero sólo PCNLAG1 es significativo, probablemente, debido al problema de multicolinealidad. Esta última regresión tiene, en comparación con las dos anteriores, el valor más alto de $r$, .99616. En conclusión, estas ecuaciones parecen ser consistentes con nociones teóricas a priori.

Tabla VII

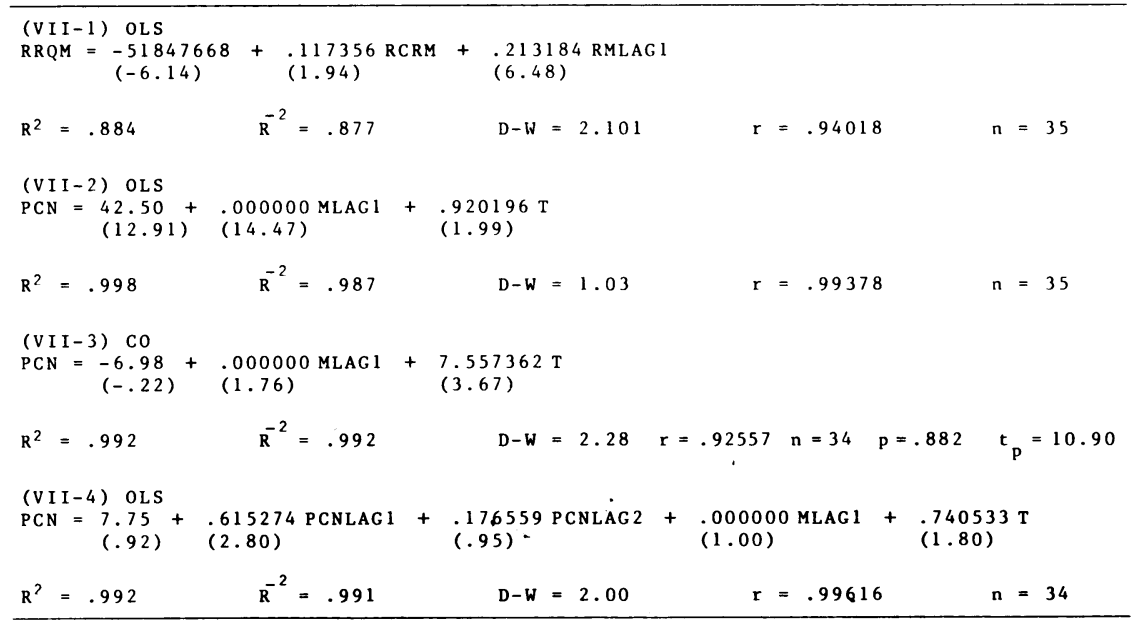

\section{Proyecciones}

En la Tabla VIII se presenta la regresión (VIII-1), que es muy parecida a la (I-5) con la diferencia de que está planteada en términos de índices. Las variables IEM, IRRQM e IRWME son índices de las variables sin la letra "I" utilizadas en las regresiones anteriores, con base 
de 100 en el primer trimestre de 1970 para las tres. Las proyecciones se realizan suponiendo que el índice de cada variable independiente (excepto T) nuevamente es de 100, pero ahora para el primer trimestre de 1979. Con distintas tasas de crecimiento de las variables independientes, se obtienen diferentes valores de IEM en el futuro, calculados a través de la regresión. Estos valores indican inmediatamente el crecimiento porcentual de EM en relación al primer trimestre de 1979.

Tabla VIII

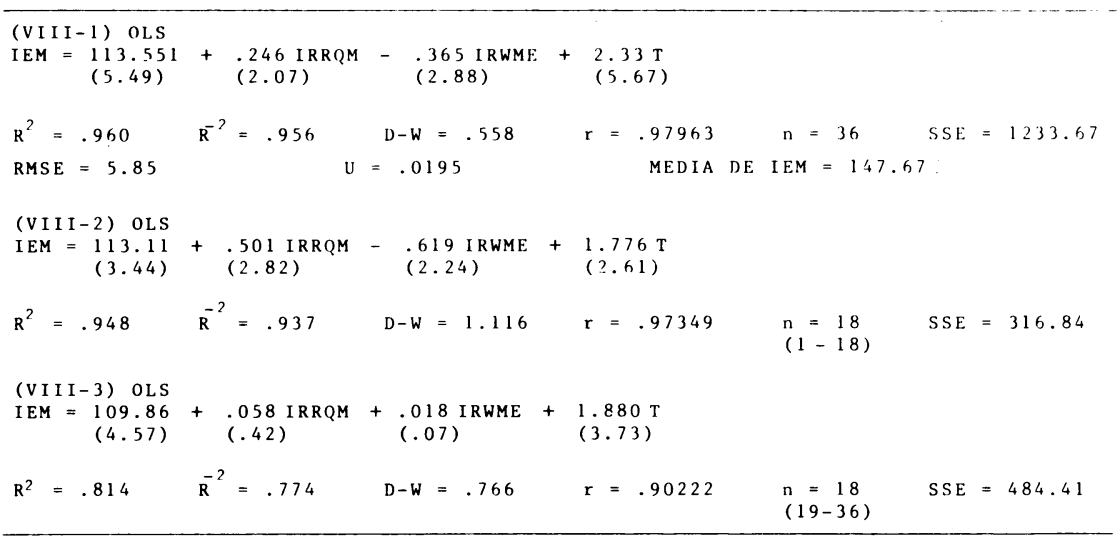

Las variables ind' rondientes de (VIII-1) tuvieron coeficientes con los signos esperados y todo fueron significativos por lo menos al 10 por ciento. En términos $c$ ? criterios de predicción, esta ecuación estimada funciona muy bien. Habíamos utilizado en las discusiones anteriores el coeficiente de correlación entre la variable dependiente predecida y la observada, o sea $r$, como nuestro indicador de la calidad predictiva de las regresiones. En esta ecuación, $r$ tuvo un valor de .97798 , que es un valor muy aceptable, como era de esperarse, ya que está basada en (I-5). Pero vale la pena considerar su poder predictivo más a fondo, ya que ésta es la regresión que genera nuestras proyecciones.

Dos estadísticos que también indican algo sobre la calidad predictiva de una regresión son, raíz del error cuadrado medio dividido por la media de la variable dependiente, y el coeficiente de desigualdad de Theil. Simbólicamente, si IEM es el índice observado, IEM* el 
índice predecido por la regresión (ambos para determinado período de tiempo), y IEM la media de IEM, la raíz del error cuadrado medio es:

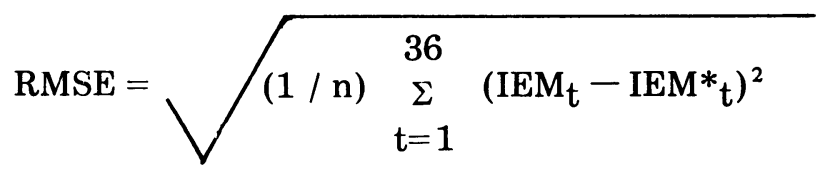

Para nuestra ecuación, RMSE $\sqrt{\text { IEM }}$ es igual a .04. Es decir, el valor del error cuadrado medio relativo de la media es pequeño, lo que es deseable en una regresión predictiva.

El propósito del coeficiente de Theil es determinar si vale la pena predecir con la regresión. La predicción más sencilla y fácil de hacer es, dar un valor de cero a la variable dependiente predecida, lo que implica ausencia de cambio en las predicciones. El coeficiente de Theil se calcula dividiendo el RMSE calculado, como se señala arriba, por el RMSE calculado en base a la predicción IEM $*=0$.

$U=\sqrt{\frac{\left({ }^{1} / \mathrm{n}\right) \Sigma\left(\mathrm{IEM}_{\mathrm{t}}-\mathrm{IEM}_{\mathrm{t}}^{*}\right)^{2}}{\left.{ }^{1} / \mathrm{n}\right) \Sigma\left(\mathrm{IEM}_{\mathrm{t}}\right)^{2}}}$

Para que la predicción econométrica valga la pena, se considera que el RMSE ordinario (o sea, el numerador) deberá ser menor que .el RMSE calculado en base a una predicción de cero (o sea, el denominador). Este criterio se resume requiriendo que $U$ sea menor que uno. Nuestra regresión tuvo un valor de U igual a .0195 y por lo tanto está incluído en el criterio por amplio margen.

Otra característica deseable, para propósitos predictivos, en una regresión empírica, es el de producir predicciones insesgadas. Se determina si el modelo realiza esto por medio de una regresión donde la variable dependiente es IEM y la independiente es IEM*. Si el coeficiente estimado de la variable independiente es igual a uno, se considera que la ecuación realiza predicciones insesgadas, ya que un aumento de una unidad en el valor de la variable predecida produce, 
en promedio, un aumento de una unidad en la variable observada. En este caso, el coeficiente tuvo exactamente el valor de uno.

Si el propósito del modelo fuera realizar predicciones a corto plazo, trimestralmente, serían importantes los puntos de transición. Bajo este criterio, si la variable dependiente observada va a aumentar de un trimestre a otro, el modelo deberá predecir un aumento, y viceversa si va a disminuir. Nuestra regresión exhibió diez errores de transición, lo cual para un período de 36 trimestres, se podría considerar como demasiado. Pero, el propósito de la regresión es predecir a mediano y largo plazo y ofrecer no sólo una predicción sino todo un escenario. Por lo tanto, en este contexto, el criterio de puntos de transición, no es importante. Pero hay otro factor de suma importancia, y la regresión (VIII-1) no luce tan favorablemente si la consideramos. Me refiero a la posibilidad de que los coeficientes del modelo no sean estables a través del tiempo.

Antes de considerar esto, veamos qué (VIII-1) podría estar mal especificado al no incluir variables con retrasos, lo que sesgaría los coeficientes estimados restantes. No obstante, al utilizar las 36 observaciones se maximiza el aprovechamiento de los datos (ya que la introducción de retrasos necesariamente reduce el tamaño disponible de la muestra), y la regresión luce favorablemente en términos de los estadísticos que resumen su poder predictivo. El aprovechamiento de todos los datos en esta regresión, permite la realización de una prueba de hipótesis sobre la estabilidad de los coeficientes a través del tiempo, maximizando también el uso de los datos disponibles. Esta prueba, como se indica enseguida, se realiza con dos "subregresiones" estimadas con 18 observaciones cada una.

Para realizar esta prueba de hipótesis se dividió la muestra en dos partes iguales, con 18 datos en cada una. Utilizando las mismas variables se corrieron también las regresiones (VIII-2) y (VII-3). Para la primera de éstas se emplearon datos del primer trimestre de 1970 al segundo trimestre de 1974 y para la segunda de estas regresiones se utilizaron datos del tercer trimestre de 1974 al cuarto trimestre de 1978. Después se procedió a probar si los coeficientes de (VII-2) y (VIII-3) son iguales o diferentes. Para entender esta prueba con mayor claridad, supongamos que la regresión de la población con 36 observaciones es:

(xiii) $\quad$ IEM $=B_{0}+B_{1}($ IRRQM $)+B_{2}($ IRWME $)+B_{3}(T), n=36$ 
mientras que las otras dos regresiones de población son:

(xiv) $\quad$ IEM $=\mathrm{C}_{0}+\mathrm{C}_{1}(\mathrm{IRRQM})+\mathrm{C}_{2}(\mathrm{IRWME})+\mathrm{C}_{3}(\mathrm{~T}), \mathrm{n}=18$

(trimestres 1-18)

(xv) $\quad$ IEM $=\mathrm{D}_{0}+\mathrm{D}_{1}(\mathrm{IRRQM})+\mathrm{D}_{2}(\mathrm{IRWME})+\mathrm{D}_{3}(\mathrm{~T}), \mathrm{n}=18$

(trimestres 19-36)

En la prueba, la hipótesis nula es

$\mathrm{H}_{0}: \mathrm{C}_{0}=\mathrm{D}_{0}, \mathrm{C}_{1}=\mathrm{D}_{1}, \mathrm{C}_{2}=\mathrm{D}_{2}, \mathrm{C}_{3}=\mathrm{D}_{3}$

y la alternativa es

$\mathrm{H}_{\mathrm{A}}: \mathrm{H}_{0}$ es falso; los coeficientes respectivos son desiguales.

El estadístico de prueba es

$$
\frac{\left(\mathrm{SSE}_{\mathrm{B}}-\mathrm{SSE}_{\mathrm{C}}-\mathrm{SSE}_{\mathrm{D}}\right) / \mathrm{K}}{\left(\mathrm{SSE}_{\mathrm{C}}+\mathrm{SSE}_{\mathrm{D}}\right) /(\mathrm{n}+\mathrm{m}-2 \mathrm{~K})} \text { distribuida como } \mathrm{F} \text { K, n }+\mathrm{m}-2 \mathrm{~K}
$$

donde SSE indica la suma de cuadrados del residuo; $\mathrm{K}$ indica el número de variables independientes más la constante (en este caso 4); $\mathrm{B}$, C y D son los subíndices para valores que surgen de las regresiones (xiii), (xiv) y (xv), respectivamente, de donde se obtienen valores de $\mathrm{SSE} ; \mathrm{n}$ y $\mathrm{m}$ son las observaciones de las regresiones (xiv) y (xv) respectivamente (en este caso ambos son iguales a 18). ${ }^{13} \mathrm{Al}$ realizar esta prueba con los resultados numéricos de las regresiones de la Tabla VIII, se rechaza $\mathrm{H}_{0}$ a un nivel de significación del 5 por ciento. Esto representa evidencia de que los coeficientes del modelo que representan estas ecuaciones no son constantes. Por lo tanto, es improbable que la estructura del modelo representado en (xiii) sea constante para el futuro, lo que indica que las proyecciones que se logran mediante su uso deben tomarse con extrema cautela.

13 Se discute esta prueba en Kmenta, págs. 373-74. Un análisis ex tensivo de este tipo de problema utilizando datos de Inglaterra se encuentra en G. Briscoe and C.J. Roberts, "Structural Breaks in Employment Functions", The Manches: School of Economic and Social Studies, March 1977, págs. 1-15. 
En la Tabla IX se presentan los escenarios que surgen combinando tasas alternativas de crecimiento de IRWME y de IRRQM, para el crecimiento de IEM en el período del puimer trimestre de 1979 al cuarto trimestre de 1982 y 1985 . El escenario más favorable de los considerados es de un crecimiento de .005 por trimestre en IRME y de .030 para IRRQM, lo que da un crecimiento en el empleo manufacturero del 46 por ciento para 1982 y del 87 por ciento para 1985 . El Programa Nacional de Empleo anticipa una tasa de crecimiento del 16 por ciento para la fuerza de trabajo en el período de 1979-1982. ${ }^{14}$ Sería lógico suponer que una meta para el crecimiento del empleo manufacturero fuera la de superar esta tasa. El escenario menos favorable de los considerados en este reporte, con un crecimiento del 21 por ciento, lo supera. Si nos guiamos por las tasas de crecimiento observadas en la muestra (cuya composición cambia a lo largo del período de estimación), el escenario que más refleja estas tasas es de un crecimiento del .005 por trimestre en IRWME y de .020 en IRRQM, lo que permite un crecimiento del empleo del 41 por ciento para 1982 y del 74 por ciento para 1985.

Tabla IX

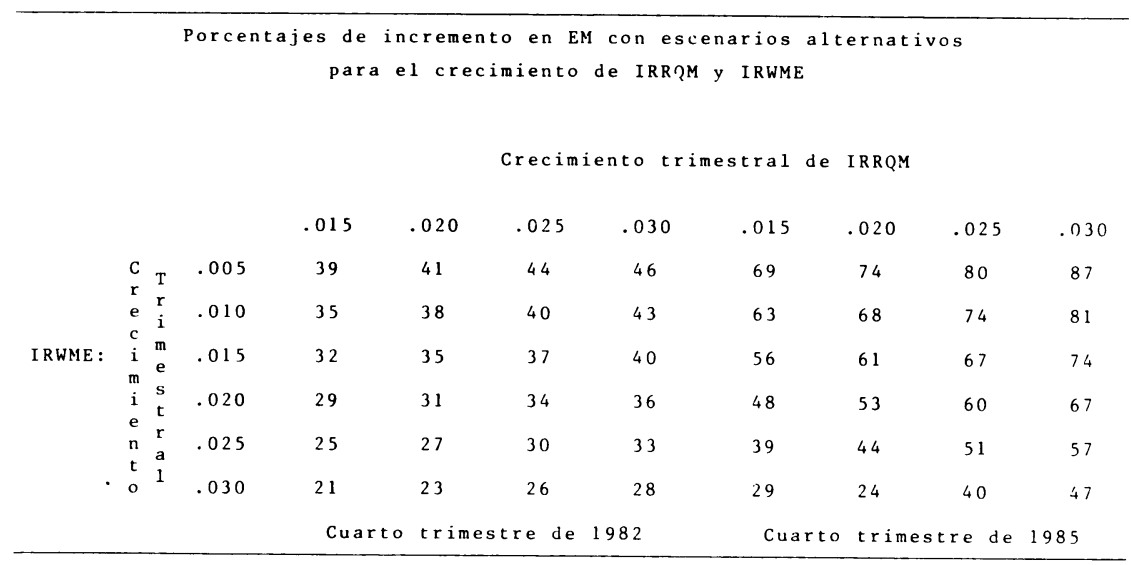

${ }^{14}$ Comisión Consultiva del Empleo, Programa Nacional de Empleo 1979-1982, Vol. III, pág. 156 , cuadro 4 . 


\section{Conclusiones}

Este estudio se ha enfocado, entre otros temas, sobre la utilización de una metodología econométrica para proyectar el crecimiento futuro del empleo manufacturero en México. Se puede argüir que esta técnica es superior al enfoque de requerimientos fijos, basados en tasas de producto-empleo, ya que explícitamente considera la posibilidad de sustitución y plantea la dependencia del empleo en las tasas del salario real. La inexistencia de datos apropiados impide la realización de proyecciones similares para la economía en su totalidad.

En términos de dar una explicación de la demanda de trabajo, este estudio no ofrece especificaciones nuevas, aunque sí añade información al acervo de conocimientos existentes, de tal manera que facilita la comparación, y los resultados son de interés para los especialistas en economía mexicana. La explicación de los salarios nominales es relativamente original, particularmente en el sentido de que se incorporan sistemáticamente los efectos de cambios en los salarios mínimos.

En relación al uso potencial de los resultados como modelo de política económica, es evidente que algunas de las variables exógenas están sujetas a control gubernamental. La forma reducida del modelo podría utilizarse como guía para la formulación de políticas, pero su cobertura limitada a un sector, desafortunadamente reduce su utilidad. No obstante, se pueden inferior implicaciones concretas. El sector manufacturero ofrece, como lo indican las proyecciones, posibilidades substanciales para el crecimiento futuro del empleo. Es uno de los sectores más dinámicos en términos del crecimiento del producto, lo que tiene un impacto positivo directo sobre el empleo. No obstante, también es un sector que podría ser susceptible de aumentos sostenidos en el salario nominal, llevando a elevadas tasas de crecimiento en la tasa de salarios reales. Al parecer, las tasas salariales reales en .manufacturas tienden a sobrepasar en promedio a las de otros sectores, los esfuerzos gubernamentales para lograr el equilibrio en los salarios entre sectores podrían servir las metas de aumentar el empleo manufacturero significativamente y mejorar la distribución del ingreso.

Limitaciones en el crecimiento de los salarios reales en esta industria podrían también, posiblemente, ahorrar divisas, que de otra manera se utilizarían en la compra de maquinaria sustitutiva de mano de obra. 


\section{LISTA DE ACTIVIDADES QUE INTEGRAN EL SECTOR MANUFACTURERO (1978, SPP)}

\section{NUMERO}

\section{ACTIVIDAD}

Preparación, conservación, empacado y enlatado de carnes

Fabricación de leche condensada, evaporada y en polvo

Preparación, conservación, empacado y envase de frutas y legumbres

Conservación, empacado y enlatado de pescados y mariscos Molienda de trigo

Fabricación de harina de maíz

Fabricación de café soluble y té

Fabricación de galletas y pastas alimenticias

Fabricación de chicles

Fabricación de almidones, féculas, levaduras y productos similares

Fabricación de aceites, margarinas y otras grasas vegetales

Fabricación de productos alimenticios para animales

Fabricación de malta

Fabricación de cerveza

Fabricación de cigarros

Hilado, tejido y acabado de algodón

Fabricación de casimires, paños, cobijas y productos similares Fabricación de estambres

Hilado, tejido y acabado de fibras artificiales

Hilado, tejido y torcido de henequén

Fabricación de triplay, tableros aglutinados y fibracel

Fabricación de pastas de celulosa y papel

Fabricación de cartón, láminas de cartón y cartoncillo

Fabricación de cajas o envases de cartón

Fabricación de llantas y cámaras

Fabricación de abonos y fertilizantes

Fabricación de fibras celulósicas y otras fibras artificiales

Fabricación de pinturas, barnices, lacas y productos similares

Fabricación de jabones, detergentes y otros productos

Fabricación de cerillos y fósforos

Regeneración de aceites lubricantes

Fabricación de coque y otros derivados del carbón mineral

Fabricación de vidrio plano, liso y labrado

Fabricación de fibra de vidrio y cristal inastillable

Fabricación de envases y ampolletas de vidrio

Fabricación de otros artículos de vidrio, incluso cristalería

Fabricación de ladrillos y tabiques refractorios y de revestimiento 


\section{NUMERO ACTIVIDAD}

3341

Fabricación de cemento hidráulico

3411

3412

3413

3421

3423

Fabricación de productos de asbesto

Fundición y laminado primaria de hierro y acero

Laminación secundaria de hierro y acero

Fabricación de tubos y postes de hierro y acero

Fundición, refinación, laminación, extracción y estiraje de cobre

Laminación, extracción y estiraje de aluminio

Fabricación de muebles y sus accesorios principalmente metálicos

Fabricación de envases y otros productos de hojalata

Fabricación de corcholatas y otros artículos troquelados

Fabricación y ensamble de maquinaria e implementos agrícolas

Fabricación y ensamble de máquinas de oficina

Fabricación de tocadiscos y receptores de radio y televisión

Fabricación de aparatos eléctricos y sus partes

Fabricación de acumuladores, baterías y pilas

Fabricación y ensamble de vehículos y automóviles

Fabricación de carrocerías para vehículos automóviles 


\section{LISTA DE VARIABLES}

Símbolo

Definición

Fuente

AQEM Producto medio nominal por persora (QM/EM)

SPP

AQHM Producto medio nominal por hora-hombre (QM/HM)

SPP

B2 a B11 Variable binarias (mudas) de salarios mínimos, cada uno correspondiendo a períodos trimestrales como sigue:

CNSM

B2: I72-IV72

B3: I73-III73

B4: IV73

B5: I74-III74

B6: IV74

B7: I75-IV75

B8: I76-III76

B9: IV76

B10: I77-IV77

B11: I78-IV78

C

Término constante

CRM Saldos nominales de crédito bancario recibidos por la industria manufacturera

BM

CUC Coeficiente porcentual de utilización de capacidad, calculado por el método "pico a pico"

DGPES

EM

Personal ocupado en manufacturas

SPP

Gastos nominales del gobierno

BM

Horas-hombre trabajadas en manufac-

SPP turas

M Saldos del medio circulante (efectivo y cheques)

BM

NWME Valor nominal por persona ocupada de los sueldos, salarios y prestaciones en la industria manufacturera (S/EM)

SPP

NWMH Valor nominal por hora-hombre de los sueldos, salarios y prestaciones en la industria manufacturera (S/HM) 
Símbolo

$\mathrm{PCN}$

QM

RCRM

RM

RRQM

RWME

RWMH

S

$\mathrm{T}$
Definición

Indice nacional de precios al consumidor (=100 en 1968)

Valor nominal de la producción manufacturera

CRM deflactado por PCN (CRM/PCN) $M$ deflactado por PCN (M/PCN) Valor real de la producción manufacturera (QM/PCN)

Valor real de los sueldos, salarios y prestaciones por persona a la industria manufacturera (S/EM) / (PCN)
Fuente

BM

SPP

BM

$\mathrm{BM}$

SPP y BM

SPP y BM

SPP y BM ciones por hora hombre en la industria manufacturera (S/HM) (PCN) Pagos saldy ial $=$ s totales (sueldos, salarios y prestaciones ) en la industria manufaturera Tiempo (=0 en I70)

SPP

\section{SIMBOLOS GENERALES USADOS EN LAS VARIABLES}

DIF primera diferencia de la variable (valor en tiempo $t$ menos valor en tiempo $t-1$ )

I Indice de la variable en cuestión (=100 en I70)

LAG1 Retraso de un período

LAG2 Retraso de dos períodos

LAG3 Retraso de tres períodos

LN Logaritmo natural

\section{FUENTES DE DATOS}

SPP Serie Estadística mensual industrial de la Secretaría de Programación y Presupuesto

BM Serie Indicadores económicos (mensual) del Banco de México

DGPES Dirección General de Política Económica y Social, SPP

CNSM Comisión Nacional de Salarios Mínimos 


\section{LISTA DE SIMBOLOS ESTADISTICOS}

Símbolo

$\mathbf{R}^{2}$

D-W

r

RMSE

U

p

$t_{p}$

SSE

OLS

CO

TSLS

TSCO

n

\section{Definición}

Coeficiente de correlación múltiple corregido por grados de libertad

Estadístico Durbin-Watson

Coeficiente de correlación entre la variable dependiente observada y la predecida por la regresión

Raíz cuadrada del error cuadrado medio

Coeficiente de Desigualdad Theil

Coeficiente de autocorrelación estimado

Valor del estadístico-t para $p$

Suma de cuadrados del residuo

Cuadrados mínimos ordinarios

Técnica iterativa de Cochrane y Orcutt

Cuadrados mínimos de dos etapas

Técnica iterativa de dos etapas

Número de observaciones. El período de estimación (trimestres y años) se relacionan con $\mathrm{n}$ de la siguiente manera:

$\mathrm{n}=36:$ I70-IV78

$\mathrm{n}=35:$ II70-IV78

$\mathrm{n}=34:$ III70-IV78

$\mathrm{n}=33:$ IV70-IV78

$\mathrm{n}=32:$ I71-IV78

$\mathrm{n}=18$ : 170 -II74 (ecuación VIII-2)

$\mathrm{n}=18$ : III74-IV78 (ecuación VIII-3)

NOTA: En las tablas, los valores del estadístico-t (valor del coeficiente dividido por su error estándar respectivo) aparecen debajo del coeficiente respectivo entre paréntesis. Todas las variables se miden de acuerdo con su magnitud, como aparecen en las fuentes de datos. En algunos casos aparecen redondeados en las fuentes citadas. 